\title{
Characterization of Rickettsia rickettsii in a Case of Fatal Brazilian Spotted Fever in the City of Rio de Janeiro, Brazil
}

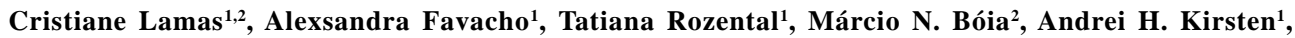 \\ Alexandro Guterres ${ }^{1}$, Jairo Barreira ${ }^{1}$ and Elba Regina S. de Lemos ${ }^{1}$ \\ ${ }^{1}$ Laboratory of Hantaviruses and Rickettsiosis (Fiocruz); ${ }^{2}$ Department of tropical Medicine, Fiocruz; Rio de Janeiro, RJ, Brazil
}

\begin{abstract}
A lethal case of Brazilian spotted fever (BSF) is presented. Clinical features were initially of gastrointestinal involvement and evolved with progression to septic shock, meningoencephalitis and death on the $6^{\text {th }}$ day of illness. Indirect immunofluorescence assay (IFA) for spotted fever group rickettsia (SFGR) was non-reactive. Diagnosis was confirmed by the polymerase chain reaction (PCR) and the nucleotide sequencing of a fragment of the ompA gene showed $100 \%$ homology to Rickettsia rickettsii. BSF has not been reported in the city of Rio de Janeiro in the last three decades, and the present description should alert the clinicians to its presence in urban Rio de Janeiro, and to the differential diagnosis with dengue fever, gastroenteritis, leptospirosis and bacterial septic shock, among others. Key-Words: Brazilian spotted fever, spotted fever group rickettsia, Rickettsia rickettsii, lethal case, Rio de Janeiro city, indirect immunofluorescence, polymerase chain reaction, central nervous system involvement.
\end{abstract}

Brazilian spotted fever (BSF) is a systemic disease caused by Rickettsia rickettsii, a bacterium transmitted by the horse tick Amblyomma cajennense. It is endemic in the Southeast of Brazil (Rio de Janeiro, São Paulo, Minas Gerais and Espírito Santo states) and affects exposed children and adults [1-4]. Case presentation mimics several conditions which are endemic in the area, such as dengue fever, leptospirosis, gastroenteritis, meningococcal meningits and severe sepsis [1-4]. This paper reports a fatal case of BSF, occurring in July, in the metropolitan area of Rio de Janeiro, with prominent sepsis, rash and neurological and cerebrospinal fluid (CSF) findings.

\section{Case Report}

A 48 year-old white male presented, six days prior to hospital admission, with acute onset of high-grade fever, myalgia, headache, nausea, vomiting and diarrhea. He was a heavy smoker, but past medical history was otherwise unremarkable. He sought medical attention and was treated symptomatically. Two days later his symptoms persisted and he developed jaundice: he was given sulfamethoxazole-trimethoprim and was sent home. The following day he sought the Emergency ward because of hematemesis and melena; he was oliguric. He was transferred to an infectious diseases reference hospital with the presumptive diagnosis of leptospirosis. He was a porter in the north area of Rio de Janeiro, and had frequent contact with rodents. He had been bitten by ticks in Campo Grande (west area of Rio Janeiro) two weeks previously, while visiting his brother, who was a horse cart driver. On arrival he was jaundiced, in deep coma with no neck stiffness, systolic Received on 18 January 2008; revised 11 April 2008.

Address for correspondence: Dr. Cristiane Lamas. Rua Presidente Carlos de Campos 81/101, Laranjeiras. Zip code: 22231-080/Rio de JaneiroRJ/Brasil. Phone number: 5521-2237-7955/Fax number: 5521- 25568455. Email: cristianelamas@gmail.com. Financial support: CAPES, Brazil.

The Brazilian Journal of Infectious Diseases 2008;12(2):149-151. (C) 2008 by The Brazilian Journal of Infectious Diseases and Contexto Publishing. All rights reserved. blood pressure was $60 \mathrm{mmHg}$, heart rate $=128 \mathrm{bpm}$, and a generalized purpuric rash was noted. He was intubated, mechanically ventilated, given rapid intravenous fluid, noradrenaline, ceftriaxone, oxacillin and chloramphenicol. Myoclonus was observed, and two hours after admission he presented a generalized tonic-clonic seizure. Initial investigation showed leukocytosis $\left(18,200\right.$ cells $/ \mathrm{mm}^{3}$, differential count: $58 \%$ polymorphs, $14 \%$ band forms), platelet count $90,000 / \mathrm{mm}^{3}$, hemoglobin $=13.9 \mathrm{~g} / \mathrm{dL}$, glucose $129 \mathrm{mg} /$ $\mathrm{dL}$, creatinine $4.4 \mathrm{mg} / \mathrm{dL}, \mathrm{AST}=314 \mathrm{IU}, \mathrm{ALT}=124 \mathrm{IU}$; blood gas analysis showed metabolic acidosis.

He died six hours after admission; post mortem lumbar puncture was performed. Cerebrospinal fluid (CSF) analysis showed 213 cells per 100 powerfields, with $70 \%$ of polymorphs, protein $>1 \mathrm{~g} / \mathrm{dL}$, glucose $=11 \mathrm{~g} / \mathrm{dL}$. Gram and Indian ink stains were normal. Latex tests for Cryptococcus neoformans, Haemophilus influenzae, Neisseria meningitidis and Streptococcus agalactiae were negative. CSF culture was negative at 48 hours; blood cultures were negative after seven days incubation.

Indirect immunofluorescence assays (IFA) for spotted fever group rickettsia (SFGR) $\left(\right.$ Panbio $\left.^{\mathrm{R}}\right)$-specific immunoglobulin $\mathrm{G}$ (IgG) antibodies were performed on serum and CSF.

Polymerase chain reaction (PCR) was performed on whole blood and serum using four previoulsy described oligonucleotide primer pairs as shown in Table 1 and Figure $1[5,6]$.

The sequence data of the PCR products were analyzed using the BLAST 2.0 program (National Center for Biotechnology Information) for homology search. The determined sequences were then analyzed for phylogenetic relationships with other sequences registered in the GenBank.

Family members of the index case, who lived where exposure to horse ticks occurred, were recalled for history, physical examination and serology for SFGR two months after the patient's death.

Ticks were captured from grass and garden near the house; they were identified taxonomically, and individually frozen at 
Table 1. Primers used for detection of spotted fever group rickettsia in a PCR assay $[5,6]$

\begin{tabular}{llc}
\hline Primer & Sequence (5'-3') & Amplicon size \\
\hline Rr190-70 & ATGGCGAATATTTCTCCAAAA & $532 \mathrm{pb}$ \\
Rr190-602 & AGTGCAGCATTCGCTCCCCCT & \\
BG1-21 & GGCAATTAATATCGCTGACGG & $650 \mathrm{pb}$ \\
BG2-20 & GCATCTGCACTAGCACTTTC & \\
RpCS877 & GGGGGCCTGCTCACGGCGG & $381 \mathrm{pb}$ \\
RpCS1258 & ATTGCAAAAAGTACAGTGAACA & \\
TZ15 & TTCTCAATTCGGTAAGGGC & $246 \mathrm{pb}$ \\
TZ16 & ATATTGACCAGTGCTATTTC & \\
\hline
\end{tabular}

Figure 1. PCR analysis of whole blood of a fatal BSF case in the city of Rio de Janeiro. Agarose gel electrophoresis, with ethidium bromide, showed the 532 bp fragment amplified with the rompA-specific set of primers (lane 2). Lane 03 - 650 bp fragment amplified with the rompB-specific set of primers. Lane 04 - 246 bp fragment amplified with the $17 \mathrm{kDa}$-protein-specific set of primers. Lane 05 - 381 bp fragment amplified with the citrate synthase-specific set of primers. Lane 06 - negative control. Lane 01 - ladder 100 bp.

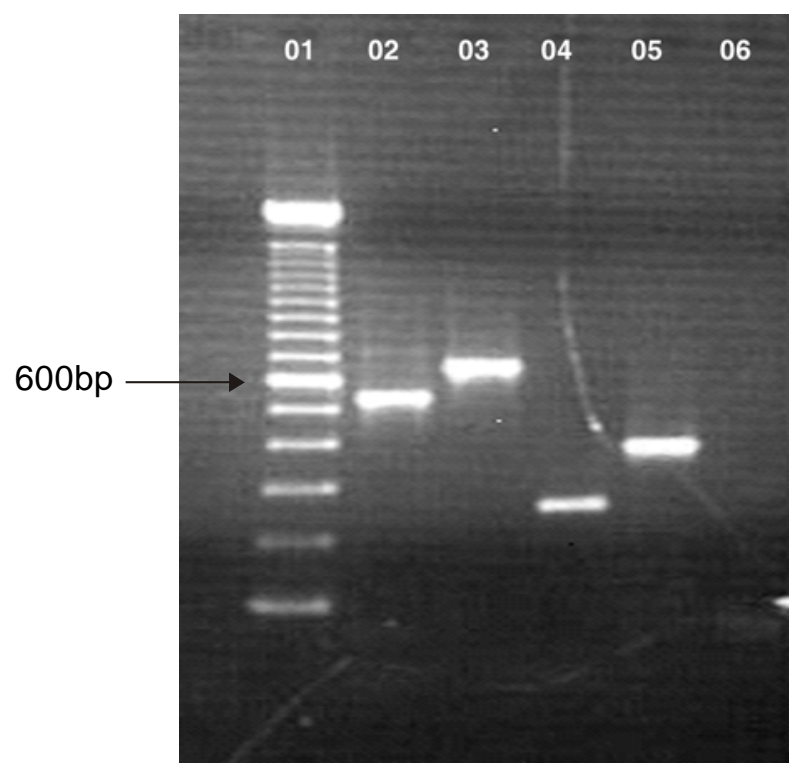

$-70^{\circ} \mathrm{C}$ until processed for DNA extraction. No ticks were collected from horses as they had been applied insecticide.

IFA for SFGR was non-reactive in the patient's serum and CSF. PCR from whole blood was positive for $R$. rickettsii and sequencing of the product amplified from the ompA gene showed $100 \%$ homology to Rickettsia rickettsii sequence available at the GenBank.

Eight family members, all of which lived in the same neighbourhood where the patient acquired his illness, were enrolled. Their ages varied between ten and 60 years None presented clinical illness in the preceding two months although all reported frequent tick bites.All had non reactive SFGR IFA.

Twenty-nine ticks (five Amblyomma cajennense, two Riphicephalus sanguineus, and 22 Anocentor nitens) were collected from grass and garden near the house. All ticks showed negative PCR results for SFGR.

\section{Discussion}

This previously healthy middle aged man presented clinical features of severe BSF following tick bites in the peak of tick reproduction, when most cases of BSF are reported. Several aspects deserve attention: he had features of a gastroenteritislike illness and was given sulfonamides, and as previously described, this probably made his outcome worse. He progressed rapidly to acute renal failure, purpuric rash, septic shock and neurological symptoms, manifested as seizure and coma. His spinal fluid analysis showed neutrophilia and low glucose levels. CSF findings in spotted fever group rickettsial disease are diverse: pleocytosis with predominant polymorphs (as in this case), predominance of eosinophils or predominance of lymphocytes. CSF may also be unremarkable [4,7-10]. The predominant cell type in CSF may be related to the timing of CSF analysis; a report on tick-borne encephalitis showed an average of 570 cells with $60 \%$ polymorphs in the first three days of illness, and a predominance of mononuclear cells from the fifth day onwards [9].

Another issue relates to jaundice: it is noted more often in BSF than in Rocky Mountain spotted fever, possibly because more adult males (with hepatitis B or hepatitis C coinfection, alcohol use or G6PD deficiency) are present in Brazilian series [3].

Chloramphenicol was started intravenously when he was admitted to hospital because there is no intravenous formulation of doxycycline in Brazil, which would be the best choice in such a severe presentation.

Another important aspect to consider is the geographic location: BSF is no longer a rural endemy, but affects inhabitants of large cities such as Rio de Janeiro and São Paulo, making it important to train doctors to think of this diagnosis, as letality is high (over $40 \%$ ) with delayed start of antibiotics. BSF is a public notifiable illness since 2001 in Brazil, and the public, including tourist agencies, need to be made aware. No cases had been reported in the city of Rio de Janeiro since 1981, although nearby towns, such as Petrópolis, sought for weekend leisure and tourism, has recently (in 2005) had an outbreak of BSF [11]. The patient's 
history of tick bites was clear, but was not elicited until he was seen at a referral hospital.

The last case reported from the city of Rio de Janeiro occurred in 1977; the report [4] accounted for two cases, both involving young males (34 and 44 years old) with cutaneous vasculitis. One of them had meningoencephalitis with abnormal CSF findings. Both were cured with intravenous chloramphenicol.

A complementary search for serological $R$. rickettsii infection in household contacts (all with tick bites) and for molecular evidence of infection in ticks from the area was performed and the results were negative: this is not surprising, because family clusters of BSF occur rarely and the prevalence of rickettsia in ticks, even in endemic areas, is low [1].

Finally, although IFA is the gold standard for diagnosis, molecular diagnosis is crucial in these rapidly fatal cases as IFA may be negative since antibody detection usually takes over ten days [12-15]. The three rickettsial reference laboratories in Brazil, located in the states of Minas Gerais, Rio de Janeiro and São Paulo, are apt to perform molecular biology tests. BSF clinical aspects and the rickettsial reference labs' abilities to make molecular diagnosis ought to be made more widely known, mainly in endemic areas where other infectious diseases as leptospirosis and dengue fever may be misdiagnosed as BSF.

\section{References}

1. Lemos E.R.S., Alvarenga F.B., Cintra M.L., et al. Rickettsial spotted fever in Brazil: a sero-epidemiological study and description of clinical cases in the State of São Paulo. Am J Trop Med Hyg 2001;65:329-34.

2. Galvão M.A., Dumler S., Mafra C.L., et al. Fatal spotted fever rickettsiosis, Minas Gerais, Brazil. Emerg Infect Dis 2003;9:1402-5.

3. Angerami R.N., Resende M.R., Feltrin A.F., et al. Brazilian spotted fever: a case series from an endemic área in southeastern Brazil: clinical aspects. Ann N Y Acad Sci 2006;1078:252-4.
4. Gonçalves A.J.R., Pinto A.N.M., Melo J.P.C., et al. Febre Maculosa Brasileira. Considerações relativas a dois casos da cidade do Rio de Janeiro. JBM 1981;41:55-7.

5. Tzianabos T., Anderson B.E., McDade J.E. Detection of Rickettsia rickettsii DNA in clinical specimens by using polymerase chain reaction technology. Clin Microbiol 1989;27:2866-8.

6. Eremeeva M., Yu X., Raoult D. Differentiation among spotted fever group rickettsiae species by analysis of restriction fragment length polymorphism of PCR-amplified DNA. Clin Microbiol 1994;32:803-10.

7. Kirk J.L., Fine D.P., Sexton D.J., Muchmore H.G. Rocky Mountain Spotted Fever. A clinical review based on 48 confirmed cases, 1943-1986. Medicine (Baltimore) 1990;69:35-45.

8. Massey E.W., Thames T., Coffey C.E., Galllis H.A. Neurologic complications of Rocky Mountain spotted fever. South Med J 1985; 78:1288-90.

9. Jeren T., Vince A. Cytologic and immunoenzymatic findings in CSF from patients with tick-borne encephalitis. Acta Cytol 1998; $42: 330-4$

10. Crennan J.M., Van Scoy R.E. Eosinophilic meningitis caused by Rocky Mountain Spotted Fever. Am J Med 1986;80:288-9.

11. Oliveira S.S., de Knegt L.V., Wildberger E.C., et al. Rickettsioses prevalence in a rural, mountainous área of Rio de Janeiro state, Brazil, 2005. In Abstracts of the $12^{\text {th }}$ International Congress on Infectious Diseases; Lisbon, Portugal; 2006 June 12-15. Int J Infect Dis 2006;10(suppl 1):S199.

12. Schattner A., Leitner M., Keysary A., Geltner D. Case report: fatal seronegative rickettsial infection diagnosed by the polymerase chain reaction. Am J Med Sci 1992;303:392-4.

13. Costa P.S., Brigatte M.E., Almeida E.P., Valle L.M.C. Atypical fulminant Rickettsia rickettsii infection (Brazilian spotted fever) presenting as septic shock and adult respiratory distress syndrome. Braz J Infect Dis 2002;6:91-6.

14. Rozental T., Eremeeva M.E., Paddock C.D., et al. Fatal case of Brazilian spotte fever confirmed by immunohistochemical staining and sequencing methods on fixed tissues. Ann N Y Acad Sci 2006;1078:257-9.

15. Nascimento E.M.M., Gehrke F.S., Maldonado R.A., et al. Detection of Brazilian spotted fever infection by polymerase chain reaction in a patient from the state of São Paulo. Mem Inst Oswaldo Cruz 2005;277-9. 